\title{
A Novel Method to Estimate the Sea State for Recycling Work on the Sea Surface
}

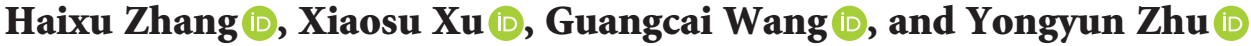 \\ Key Laboratory of Micro-Inertial Instrument and Advanced Navigation Technology, Ministry of Education, \\ School of Instrument Science and Engineering, Southeast University, Nanjing, China \\ Correspondence should be addressed to Xiaosu Xu; xxs@seu.edu.cn
}

Received 2 December 2020; Revised 28 December 2020; Accepted 3 January 2021; Published 18 January 2021

Academic Editor: Gaoge $\mathrm{Hu}$

Copyright (C) 2021 Haixu Zhang et al. This is an open access article distributed under the Creative Commons Attribution License, which permits unrestricted use, distribution, and reproduction in any medium, provided the original work is properly cited.

The recycling of marine exploration equipment after it has surfaced is greatly affected by sea state. In order to estimate the sea state in real time, this paper proposes a method for measuring wave elevation, which modifies the integrated results of GNSS/SINS in the up direction by virtual horizontal lines to extract wave fluctuation information. From these wave information, the significant wave heights (SWH) can be calculated as the only input parameter of P-M spectrum, and a series of wave height data can be further simulated. When the GNSS is interrupted due to severe sea state, the simulated data can be integrated with the SINS to deal with the data distortion problem. The simulation results show that the application of wave spectrum in the GNSS intermittent situation has obvious improvement effect and important significance.

\section{Introduction}

From the experience of engineering practice, the recycling of marine detection equipment is often carried out in the good sea state, as shown in Figure 1, because the safety of the crane operation will be seriously threatened by the shaking of the ship in the bad sea state. Although the recycling work can be arranged in advance according to weather forecast, the complex sea state still cannot be estimated very well, especially the impact of swell $[1,2]$. Missing the best recycling time may cause the device to run out of power, lose contact, and disappear into the sea. Therefore, the acquisition of realtime sea state information is of great significance in the process of equipment recycling.

In order to obtain wave elevation data under different application backgrounds, many scholars have carried out research studies on it. Using buoys to collect wave data is the most common and effective method. Crosby et al. [3] provide a new method for estimating offshore boundary conditions according to the buoy observations at 16 near-shore buoy sites. Buoys are also used to validate Haiyang-2A altimeter SWH data [4 and 5]. In addition to buoys, high-frequency surface wave radar, X-band marine radar, and satellite can also work for wave data acquisition [6-9].
However, for detection equipment such as AUVs and underwater gliders, their position after surfacing is uncertain beforehand, and buoys cannot be preset to measure the sea conditions around them. The use of satellite or radar to measure wave heights requires additional hardware support. As the only subsystem that can sense wave elevation that they are equipped with, the strap-down inertial navigation system (SINS) cannot be simply used to obtain the sea state due to its error accumulation.

The SINS is irreplaceable in the aspect of navigation with the restrictions of other sensors because of its high precision in short time [10-13]. Especially, the combination of the SINS and GNSS has been widely used [14-19], which not only limits the divergence of the SINS but also can provide high-positioning accuracy. If applied to the detection of wave elevation on the sea surface in the up direction, the following two problems need to be solved:

(1) The error of the integrated method is too large, which covers the real wave elevation information

(2) Limited by the output frequency of the GNSS, the real-time performance of the results needs to be improved 


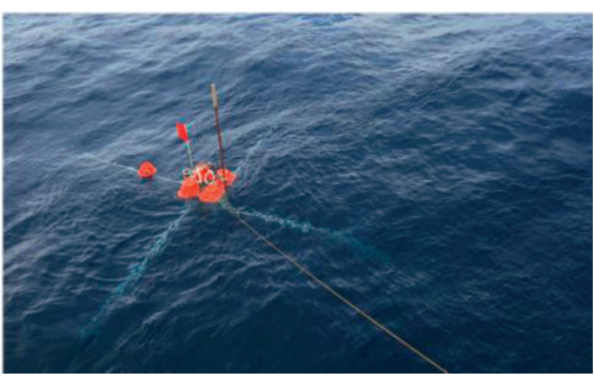

FIgURE 1: Equipment to be recycled in the good sea state.

In order to solve the above problems and provide the real-time sea state of the target equipment, a novel fusion method is proposed to estimate the sea surface wave elevation. In this way, the crew can get real-time information about the sea conditions around the ocean detection equipment to avoid working under bad sea conditions and arrange the recycling work reasonably. The method makes full use of the high precision of the SINS in short time to sensitive wave characteristic and establishes the wave model of current time to fuse information in the next moment.

The remainder of the paper is organized as follows. The mathematical model is described in Section 2. Section 3 introduces the fusion about SINS, GNSS, and simulated waves. The simulations and semiphysics experiment are discussed in Section 4. Finally, Section 5 concludes this paper.

\section{System Model}

2.1. SINS State Equation. The physical parameters measured by gyroscopes and accelerometers of the SINS are used to calculate the trajectory of the carrier in the up direction when floating on the sea surface. The state equation is given as follows according to Wang et al [20]:

$$
\dot{X}=F X+G W
$$

where the state vector can be expressed as follows:

$$
X=\left[\phi_{x} \phi_{y} \phi_{z} \delta V_{E}^{n} \delta V_{N}^{n} \delta V_{U}^{n} \delta \lambda \delta L \delta h \varepsilon_{x} \varepsilon_{y} \varepsilon_{z} \nabla_{x} \nabla_{y} \nabla_{z}\right]^{T},
$$

where $\phi_{x}, \phi_{y}$, and $\phi_{z}$ denote misalignment angles, $\delta V_{E}^{n}, \delta V_{N}^{n}$, and $\delta V_{U}^{n}$ denote velocity errors, $\delta \lambda, \delta L$, and $\delta h$ denote the longitude, latitude, and height errors, respectively, $\varepsilon_{x}, \varepsilon_{y}$, and $\varepsilon_{z}$ denote gyroscope biases in three directions of the frame $b$, and $\nabla_{x}, \nabla_{y}$, and $\nabla_{z}$ denote accelerometer biases.

The state transition matrix $\mathbf{F}$ is expressed as follows:

$$
\mathbf{F}=\left[\begin{array}{lllll}
\mathbf{M}_{\mathbf{a a}} & \mathbf{M}_{\mathrm{av}} & \mathbf{M}_{\mathrm{ap}} & -\mathbf{C}_{\mathbf{b}}^{\mathbf{n}} & 0_{3 \times 3} \\
\mathbf{M}_{\mathbf{v a}} & \mathbf{M}_{\mathbf{v v}} & \mathbf{M}_{\mathbf{v p}} & 0_{3 \times 3} & \mathbf{C}_{\mathbf{b}}^{\mathbf{n}} \\
0_{3 \times 3} & \mathbf{M}_{\mathbf{p v}} & \mathbf{M}_{\mathbf{p p}} & 0_{3 \times 3} & 0_{3 \times 3} \\
0_{6 \times 3} & 0_{6 \times 3} & 0_{6 \times 3} & 0_{6 \times 3} & 0_{6 \times 3}
\end{array}\right],
$$

where direction cosine matrix $\mathbf{C}_{\mathbf{b}}^{\mathbf{n}}$ denotes the rotational transform from the $b$-frame to the $n$-frame. And, we have

$$
\begin{aligned}
& \mathbf{M}_{\mathbf{a a}}=\left[\begin{array}{ccc}
0 & \omega_{i e} \sin L+\frac{V_{E}}{R_{N}+h} \tan L-\omega_{i e} \cos L-\frac{V_{E}}{R_{N}+h} \\
-\omega_{i e} \sin L-\frac{V_{E}}{R_{N}+h} \tan L & 0 & -\frac{V_{N}}{R_{M}+h} \\
\omega_{i e} \cos L+\frac{V_{E}}{R_{N}+h} & \frac{V_{N}}{R_{M}+h}
\end{array}\right], \\
& \mathbf{M}_{\mathbf{a v}}=\left[\begin{array}{ccc}
0 & -\frac{1}{R_{M}+h} & 0 \\
\frac{1}{R_{N}+h} & 0 & 0 \\
\frac{\tan L}{R_{N}+h} & 0 & 0
\end{array}\right] \\
& \mathbf{M}_{\text {ap }}=\left[\begin{array}{ccc}
0 & 0 & \frac{V_{N}}{\left(R_{M}+h\right)^{2}} \\
-\omega_{i e} \sin L & 0 & -\frac{V_{E}}{\left(R_{N}+h\right)^{2}} \\
\omega_{i e} \cos L+\frac{V_{E} \sec ^{2} L}{R_{N}+h} & 0 & -\frac{V_{E} \tan L}{\left(R_{N}+h\right)^{2}}
\end{array}\right],
\end{aligned}
$$




$$
\begin{aligned}
& \mathbf{M}_{\mathrm{va}}=\left[\begin{array}{rcc}
0 & -\mathbf{f}_{\mathrm{U}}^{\mathbf{n}} & \mathbf{f}_{\mathrm{N}}^{\mathbf{n}} \\
\mathbf{f}_{\mathrm{U}}^{\mathbf{n}} & 0 & -\mathbf{f}_{\mathrm{E}}^{\mathbf{n}} \\
-\mathbf{f}_{\mathrm{N}}^{\mathbf{n}} & \mathbf{f}_{\mathrm{E}}^{\mathbf{n}} & 0
\end{array}\right], \\
& \mathbf{M}_{\mathbf{v v}}=\left[\begin{array}{ccc}
\frac{V_{N} \tan L-V_{U}}{R_{N}+h} & 2 \omega_{i e} \sin L+\frac{V_{E} \tan L}{R_{N}+h}-2 \omega_{i e} \cos L-\frac{V_{E}}{R_{N}+h} \\
-2 \omega_{i e} \sin L-\frac{2 V_{E} \tan L}{R_{N}+h} & -\frac{V_{U}}{R_{M}+h} & -\frac{V_{N}}{R_{M}+h} \\
2 \omega_{i e} \cos L+\frac{2 V_{E}}{R_{N}+h} & \frac{2 V_{N}}{R_{M}+h} & 0
\end{array}\right] \text {, } \\
& \mathbf{M}_{\mathrm{vp}}=\left(\mathbf{V}^{\mathbf{n}} \times\right)\left(2 \mathbf{M}_{1}+\mathbf{M}_{3}\right)+\mathbf{M}_{4}, \\
& \mathbf{M}_{\mathbf{1}}=\left[\begin{array}{ccc}
0 & 0 & 0 \\
-\omega_{i e} \sin L & 0 & 0 \\
\omega_{i e} \cos L & 0 & 0
\end{array}\right], \\
& \mathbf{M}_{3}=\left[\begin{array}{ccc}
0 & 0 & \frac{V_{N}}{\left(R_{M}+h\right)^{2}} \\
0 & 0 & -\frac{V_{E}}{\left(R_{N}+h\right)^{2}} \\
\frac{V_{E} \sec ^{2} L}{R_{N}+h} & 0 & -\frac{V_{E} \tan L}{\left(R_{N}+h\right)^{2}}
\end{array}\right] \\
& \mathbf{M}_{4}=\left[\begin{array}{ccc}
0 & 0 & 0 \\
0 & 0 & 0 \\
-g_{e} \sin 2 L\left(\beta-4 \beta_{1} \cos 2 L\right) & 0 & \beta_{2}
\end{array}\right], \\
& \mathbf{M}_{\mathbf{p v}}=\left[\begin{array}{ccc}
0 & \frac{1}{R_{M}+h} & 0 \\
\frac{\sec L}{R_{N}+h} & 0 & 0 \\
0 & 0 & 1
\end{array}\right] \text {, } \\
& \mathbf{M}_{\mathbf{p p}}=\left[\begin{array}{ccc}
0 & 0 & -\frac{V_{N}}{\left(R_{M}+h\right)^{2}} \\
\frac{V_{E} \sec L \tan L}{R_{N}+h} & 0 & -\frac{V_{E} \sec L}{\left(R_{N}+h\right)^{2}} \\
0 & 0 & 0
\end{array}\right],
\end{aligned}
$$

where $\omega_{\text {ie }}$ is the rotational angular velocity of the Earth, $L$ and $h$ are the position components in latitude and altitude, $V_{E}, V_{N}$, and $V_{U}$ are the velocity components in the east, north, and up directions, $\mathbf{f}_{\mathbf{N}}^{\mathbf{n}}, \mathbf{f}_{\mathrm{E}}^{\mathbf{n}}$, and $\mathbf{f}_{\mathbf{U}}^{\mathbf{n}}$ are the measurements of specific force in the east, north, and up directions, $g_{e}$ is the acceleration of gravity, $R_{M}$ and $R_{N}$ are the radii of curvature in meridian and prime vertical [21], $\beta$ is gravity flattening, and $f$ is flattening of ellipsoid, and we have 


$$
\mathbf{G}=\left[\begin{array}{cc}
-\mathbf{C}_{\mathbf{b}}^{\mathbf{n}} & 0_{3 \times 3} \\
0_{3 \times 3} & \mathbf{C}_{\mathbf{b}}^{\mathbf{n}} \\
0_{9 \times 3} & 0_{9 \times 3}
\end{array}\right] .
$$

And, the noise vector $\mathbf{W}$ is written as follows:

$$
\mathbf{W}=\left[\begin{array}{c}
\mathbf{w}_{\mathrm{g}}^{\mathbf{b}} \\
\mathbf{w}_{\mathbf{a}}^{\mathbf{b}}
\end{array}\right],
$$

where $\mathbf{w}_{\mathrm{g}}^{\mathbf{b}}$ and $\mathbf{w}_{\mathbf{a}}^{\mathbf{b}}$ denote angular velocity measurement noise of gyroscopes and specific force measurement noise of accelerometers, respectively.

2.2. Measurement Equation. Satellite positioning is very effective in dealing with the divergence of the SINS, so the GNSS in the detector can be used to correct the accumulated errors in the up direction. The measurement equation of the GNSS/SINS integrated method is defined as follows:

$$
\mathbf{Z}=\mathbf{H X}+\mathbf{V},
$$

where the measurement vector is expressed as follows:

$$
\mathrm{Z}=\left[h_{\mathrm{SINS}}-h_{\mathrm{GNSS}}\right],
$$

where $h_{\text {SINS }}$ and $h_{\text {GNSS }}$ denote the measurements in the up direction of the SINS and GNSS, respectively.

The observation matrix $\mathbf{H}$ and observation error matrix $\mathrm{V}$ are expressed as follows:

$$
\begin{aligned}
& \mathbf{H}=\left[\begin{array}{lll}
0_{1 \times 8} & I_{1 \times 1} & 0_{1 \times 6}
\end{array}\right], \\
& \mathbf{V}=\left[\begin{array}{l}
v_{z}
\end{array}\right],
\end{aligned}
$$

where the variable $v_{z}$ is the measurement error of the GNSS in the up direction. Although corrected by the GNSS, the error is still unacceptable relative to the wave heights.

\section{Improved Method for Estimating Sea State}

The solution of the SINS in the up direction represents the characteristic of the target moving along with waves on the sea surface; thus, it can provide a reference for staffs to know the sea state around the remote target. However, the SINS will diverge quickly in the up direction without any other sensors to perform correction. The GNSS plays a certain role in the correction of the SINS, but considering its own error and real-time performance, the results obtained by means of the integrated method are still not enough to represent the characteristics of wave heights. Therefore, we need to find another way to extract the waveform from the existing conditions.

3.1. Corrected Waves from SINS/GNSS. The SINS can observe the wave heights with good precision and high frequency. However, the wave heights observed by the SINS in the up direction always deviate from the horizontal line due to error accumulation, as shown in Figure 2.

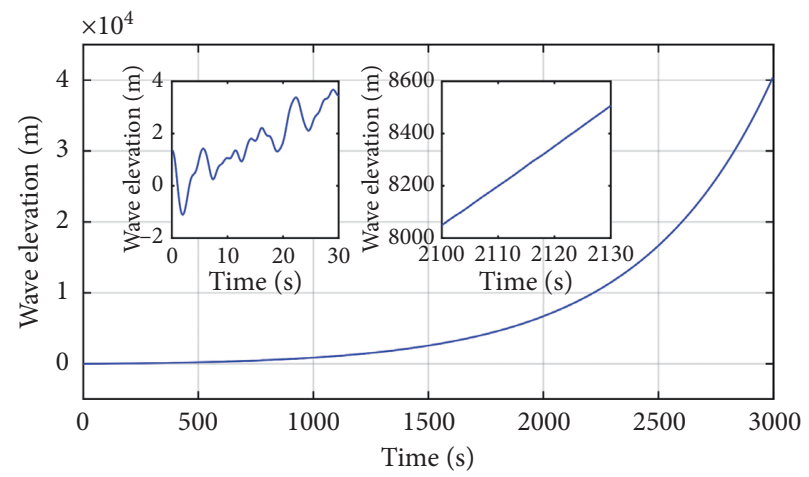

Figure 2: The time history curve of wave elevation observed by the SINS.

Through the comparison in Figure 2, we can also get that the wave fluctuation is retained in the initial period of time, while gradually it disappears in the following time. Therefore, in order to obtain wave information, the divergence problem must be solved first.

The combination of the GNSS and SINS is a classic way to weigh divergence and accuracy in navigation. It is also effective in solving the positioning problem in the up direction, as shown in Figure 3. However, although the divergence problem is solved, the magnitude of the integrated method errors is still unacceptable relative to the wave heights.

Waves always float about the horizontal line up and down, but the error accumulation of the SINS seems to make the horizontal line no longer horizontal. To obtain the heights more accurately, a simulation of the virtual horizontal line for the waves in the data fragment is needed first, as shown in Figure 4.

In the data segment with the virtual horizontal line as the reference, different from the observed value $h$, wave height $v h$ is the difference value in the up direction in a short time, and the problem of error accumulation can be less concerned. In addition, $t_{1}$ and $t_{2}$ are the time corresponding to the measured wave heights, while $v t_{1}$ and $v t_{2}$ are to the virtual horizontal line. Therefore, the trajectory of the waves can be corrected after finding the virtual horizontal line. The least squares method is an effective tool to solve this problem, whose minimum mean square error function can be expressed as follows:

$$
\mathbf{J}(\boldsymbol{\theta})=(\mathbf{B}-\mathbf{T} \boldsymbol{\theta})^{T}(\mathbf{B}-\mathbf{T} \boldsymbol{\theta}),
$$

where

$$
\begin{aligned}
& \mathbf{B}=\left[h_{1} h_{2} \cdots h_{n}\right]^{T}, \\
& \mathbf{T}=\left[\begin{array}{cc}
t_{1} & 1 \\
\vdots & \vdots \\
t_{n} & 1
\end{array}\right], \\
& \boldsymbol{\theta}=\left[\begin{array}{c}
a \\
b
\end{array}\right],
\end{aligned}
$$




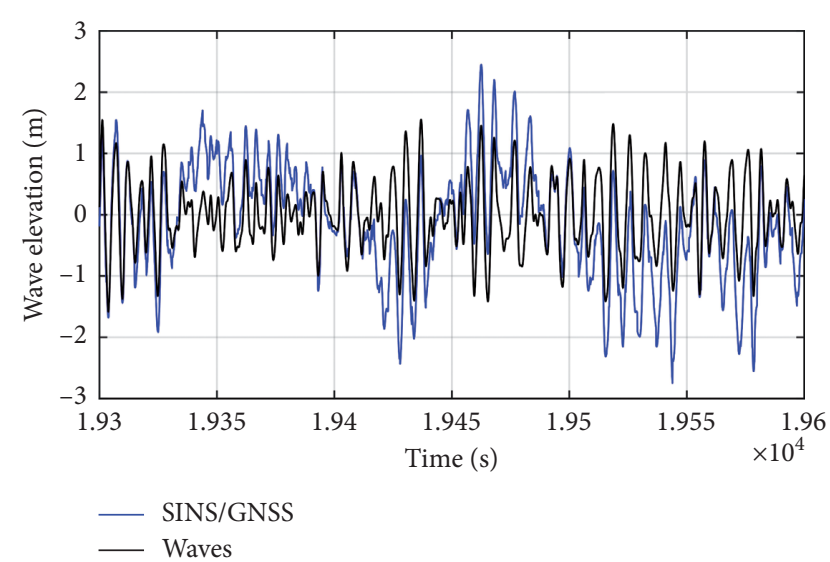

Figure 3: Comparison of the integrated results and true values in the up direction.

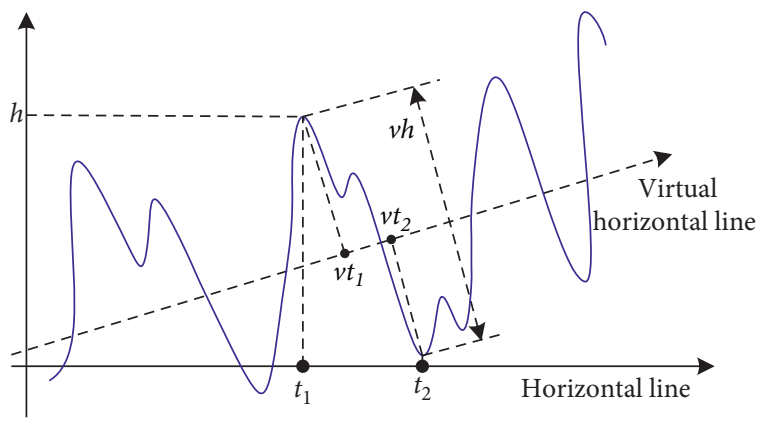

FIgURE 4: The schematic diagram of the virtual horizontal line.

where $n$ is the number of measured values in a data segment. The virtual horizontal line can be approximated as follows:

$$
h=a t+b .
$$

Waves can be considered as the result of the superposition of countless random simple cosine waves, so the average height of the resultant wave surface is zero. According to this principle, the sum of all wave heights to the virtual horizontal line should also be zero. Considering the errors, the sum of wave heights can be denoted as follows:

$$
\operatorname{SUM}\left(d_{1} \cdots d_{i} \cdots d_{n}\right)=\varepsilon
$$

where $d_{i}$ is the distance from the $i$ th observed wave height to the virtual horizontal line, whose sign depends on the position relative to the virtual horizontal line. In addition, $\varepsilon$ comes from system errors. Then, the sample values referring to the true horizontal line can be corrected as follows:

$$
c h_{i}=d_{i}-\frac{\varepsilon}{n}
$$

The corrected sample value $c h_{i}$ can be used to get the wave information referring to the real horizontal line.
3.2. Estimating Waves by P-M Spectrum. The above method to calculate wave heights is based on the SINS and GNSS integrated method in good condition. However, GNSS signal interruption is not rare for the detector floating on the sea surface in the harsh marine environment. In order to extract the wave data more accurately under finite conditions, the model of wave mechanics for ocean engineering is introduced in this paper.

Waves are unpredictable due to the influence of many factors. Although wave elevation is a random value that changes with time, it can still be characterized by mathematical simulation due to its stationarity and ergodic property of various states [22]. As a single input parameter spectrum, the P-M spectrum, according to Shouhua et al. [23], can be used for wave elevation simulation based on the acquired SWH by the SINS, which is defined as follows:

$$
S(\omega)=A \omega^{-5} \exp \left(-B \omega^{-4}\right),
$$

where

$$
\begin{aligned}
& A=\alpha g^{2}, \\
& B=0.74\left(\frac{g}{V_{19.4}}\right)^{4} .
\end{aligned}
$$

Phillips constant $\alpha$ in the formula above is defined as follows:

$$
\alpha=8.1 \times 10^{-3} .
$$

The wave spectrum in equation (30) contains only one parameter, namely, $V_{19.4}$, which represents the wind speed at 19.4 meters above the sea surface. However, IMU cannot provide wind speed information, so further conversion is needed.

According to the following definition of the average wave height of partial big waves,

$$
H_{1 / p}=\left(8 m_{0} \ln p\right)^{1 / 2}+p\left(2 \pi m_{0}\right)^{1 / 2}\left\{1-\operatorname{erf}\left[(\ln p)^{1 / 2}\right]\right\} .
$$

We obtain the average wave elevation of the first third of the waves as follows:

$$
H_{1 / 3}=H_{s} \approx 4.0\left(m_{0}\right)^{1 / 2},
$$

where $H s$ represents the SWH and $m_{0}$ is the zeroth moment of the spectrum expressed as follows:

$$
m_{0}=\frac{A}{4 B} \text {. }
$$

The value $H_{s}$ can be approximated as below according to equations (35) and (36):

$$
H_{s} \approx 0.209 \frac{V_{19.4}^{2}}{g} .
$$


It can be seen that the wave elevation is proportional to the square of the wind speed, so the wave spectrum represented by the single parameter of the wave elevation can be written as follows:

$$
S(\omega)=\frac{0.78}{\omega^{5}} \exp \left(-\frac{3.11}{\omega^{4} H_{s}^{2}}\right) .
$$

The constituent waves of high frequency and small frequency provide little energy, and the bulk of the energy comes from the constituent waves with the narrow band. If the part of the total energy is allowed to be omitted on the high and low sides of the spectrum, the upper and lower bounds of frequency of the constituent waves can be written as follows:

$$
\begin{aligned}
\omega_{L} & =\left(-\frac{3.11}{H_{s}^{2} \ln \mu}\right)^{1 / 4}, \\
\omega_{H} & =\left(-\frac{3.11}{H_{s}^{2} \ln (1-\mu)}\right)^{1 / 4} .
\end{aligned}
$$

In engineering applications, the time history of a series of irregular waves can be simulated by the target spectrum after getting the characteristic wave parameters. After taking P-M spectrum as the target spectrum and dividing the total energy into $N$ parts according to the equipartition energy method, the waves' time history can be expressed as follows:

$$
\eta(t)=\left(\frac{2 m_{0}}{N}\right)^{1 / 2} \sum_{i=1}^{N} \cos \left(\widehat{\omega}_{i} t+\varphi_{i}\right),
$$

where

$$
\widehat{\omega}_{i}=\frac{\left(\omega_{i}+\omega_{i-1}\right)}{2}
$$

In addition, the value $\varphi_{i}$ is the initial phase uniformly distributed in the interval of $[0,2 \pi]$, while $\omega_{i}$ is the boundary frequency defined as follows:

$$
\omega_{i}=\left[\frac{B}{\ln (N / i)}\right]^{1 / 4} .
$$

According to equations (38) and (41), a part of the waves can be simulated, as shown in Figure 5, with SWH of 3 meters as the only parameter of P-M spectrum.

The waves simulated by the spectrum satisfy the stationarity and ergodicity of the general waves statistically, which fluctuates around the horizontal line, and the mean value is close to zero. As long as the SWH of the previous moment is collected, the approximate value of the wave heights in the next short moment can be estimated by this method.

Compared with other spectra, $\mathrm{P}-\mathrm{M}$ spectrum is more suitable for the engineering application proposed in this paper because its only input parameter is more easy to be obtained by the SINS. However, the integral results of the SINS will diverge without the correction of the GNSS, which

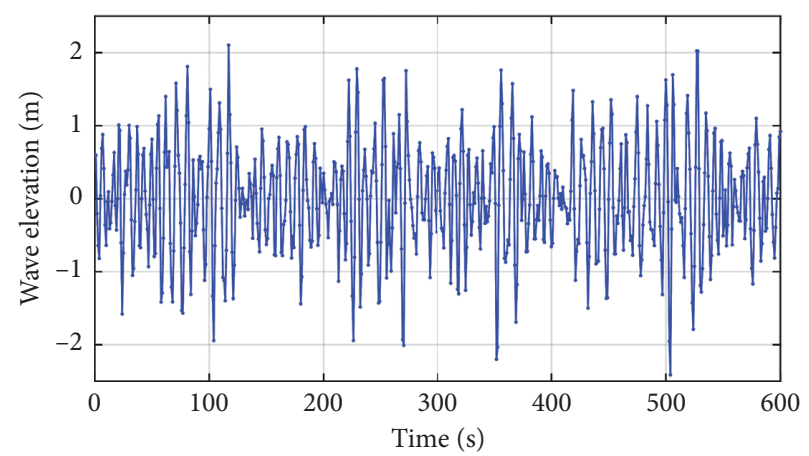

Figure 5: Wave simulation by P-M spectrum.

indirectly determines that the wave heights simulated by P-M spectrum cannot be applied independently.

3.3. Get Wave Heights with Intermittent GNSS. It is not uncommon for the surfaced detection equipment to lose GNSS signals in the bad sea state. Without effective correction, the divergence of the SINS in the up direction will weaken its ability to extract wave features, as shown in Figure 6.

As can be seen from the above figures, (a) is the measurement results at the beginning of the intermittent state, while (b) represents that the state lasted for a period. Although the measurement effect is good at the beginning, as time goes by, the divergence of the SINS cannot be effectively corrected, and the wave height data calculated according to equation (29) gradually distorts until the characteristics of wave heights cannot be recognized at last.

In order to improve this problem, we tried to use wave spectrum to simulate wave height data to assist the SINS to maintain a considerable height measurement in the intermittent GNSS state. The only input parameter of P-M spectrum is SWH according to equation (38). Therefore, it is necessary to analyze the latest measured data fragment to calculate SWH before the GNSS signal is interrupted. After obtaining the corrected wave height information by equation (29), the zero-up crossing method, as shown in Figure 7, is used to calculate wave heights, where time $t_{1}$ and $t_{2}$ are the zero-up crossing points and $h_{1}$ and $h_{2}$ are the wave heights, respectively.

After taking the average wave elevation of the first third of the waves as SWH, the wave elevation can be immediately simulated by $\mathrm{P}-\mathrm{M}$ spectrum as follows according to equation (41):

$$
\eta(t)=\left\{\eta_{1} \eta_{2} \eta_{3} \ldots \eta_{n}\right\}
$$

where the time interval can be set small enough to obtain a suitable sampling frequency to match the SINS. In addition, the measurement equation of the SINS and simulated wave elevation integrated method is defined as follows:

$$
\mathrm{Z}_{\mathrm{s}}=\mathbf{H X}+\mathrm{V}_{\mathrm{s}}
$$




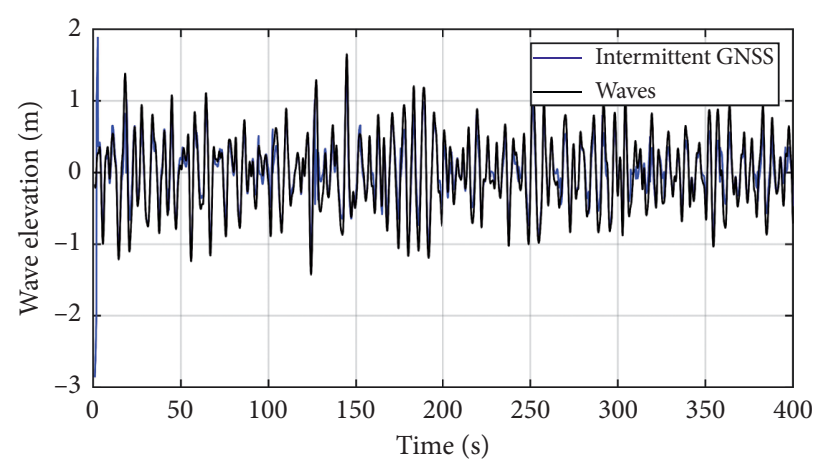

(a)

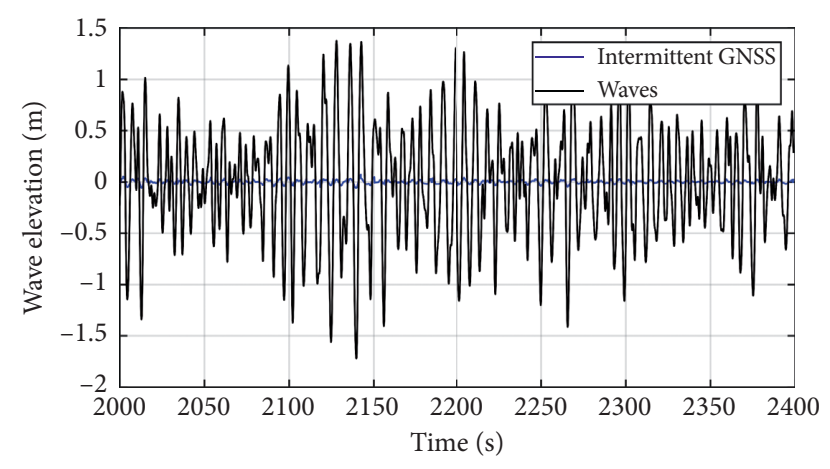

(b)

Figure 6: The wave height results with intermittent GNSS signals.

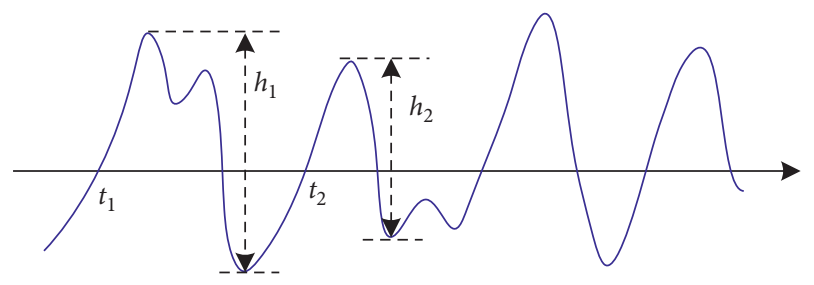

FIGURE 7: The wave height results with intermittent GNSS signals.

where the observation error matrix $\mathbf{V}_{\mathbf{s}}$ represents the error of the simulated wave elevation, and the measurement vector is expressed as follows:

$$
\mathbf{Z}_{\mathrm{s}}=\left[h_{\mathrm{SINS}}-\eta(t)\right]
$$

The integrated method can fill the gap of GNSS interruption in bad sea conditions. In addition, under the correction of the connected GNSS, the wave elevation information in the environment of the surface detection equipment can be output to provide the sea state information continuously and stably.

\section{Experiment and Simulation}

In order to demonstrate the effectiveness of the integrated method by wave spectrum for the surfaced detection equipment to estimate the sea state, experiment and simulation will be carried out to verify the following aspects:

(1) Advantages of P-M spectrum in wave elevation simulation

(2) The performance of the virtual horizontal line in wave elevation extraction

(3) The response effect of the novel integrated method to GNSS interruption

Therefore, each experiment or simulation is carried out in the following order.

4.1. P-M Spectrum for Simulating Wave Elevation. Before using wave spectrum to integrate the SINS in the up direction, it is necessary to analyze the statistical characteristic of simulated waves to verify its feasibility. A set of wave elevation data collected in the offshore area of the Yellow Sea in China is selected as the reference sample to verify the advantages of wave spectrum in wave elevation simulation. The comparisons of true and simulated waves (SW) are shown in Figure 8.

Since the waves can be regarded as a random process, the simulation based on the wave spectrum cannot reproduce the motion trajectory, but it can be used to approximate the true values statistically. Furthermore, 100 simulations were carried out according to the same series of true waves, and the statistical parameters are shown in Figure 9.

The waves could be regarded as the result of superposition of an infinite number of random simple cosine waves, so the average height of the waves should be zero in theory. In this respect, wave simulation works well as shown above. The variance of wave elevation can be regarded as the wave energy. When the waves are in a stable stage, the variance changes slowly. Limited by the sampling frequency of the wave buoy, the variance of the true values in Figure 9 is slightly higher than the simulated values. At the same time, the sensitivity of wave simulation to $\mathrm{SWH}$ is within the acceptable range. In general, the simulated waves have good statistical characteristics, and more importantly, their sampling frequency is not limited, which will make their positioning assistance in the up direction more meaningful.

4.2. Advantage of the Virtual Horizontal Line in the UP Direction. After verifying the feasibility of wave spectrum simulation, the motion state simulation of the target in the direction of up floating on the sea surface is shown in Figure 10. The target moves up and down according to the trajectory of wave elevation, with uniform distribution of velocity and acceleration, which conforms to the characteristics of the stable wave motion.

The positioning results of the GNSS/SINS in the up direction are shown in Figure 11. It can be seen that, with the help of the GNSS, the divergence of the SINS in the up direction is solved, but it is still not enough to represent the characteristic of wave elevation.

In view of the problem in Figure 11, the virtual horizontal line of the original data was calculated according to 


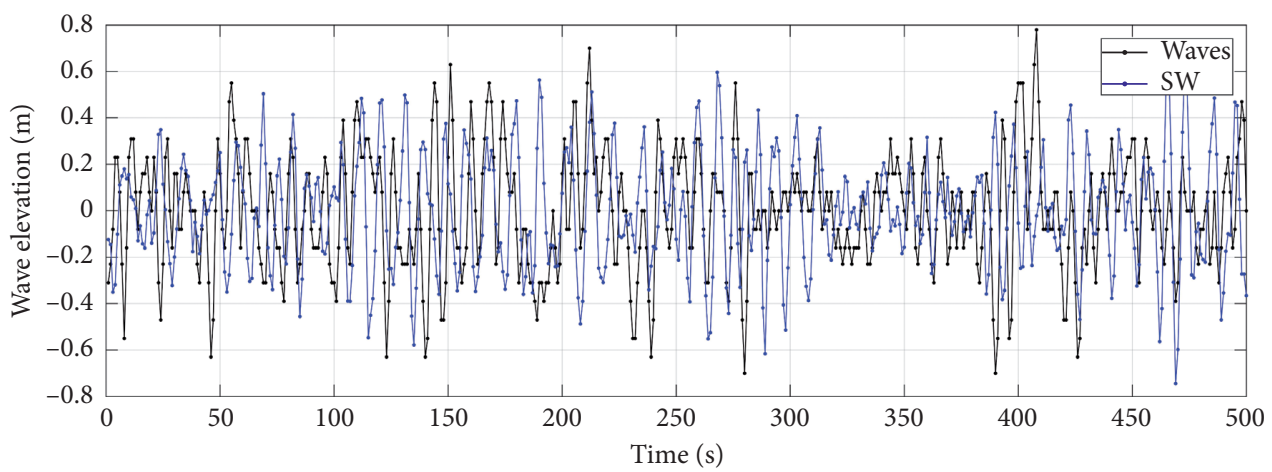

Figure 8: Comparison of true and simulated values of wave elevation.
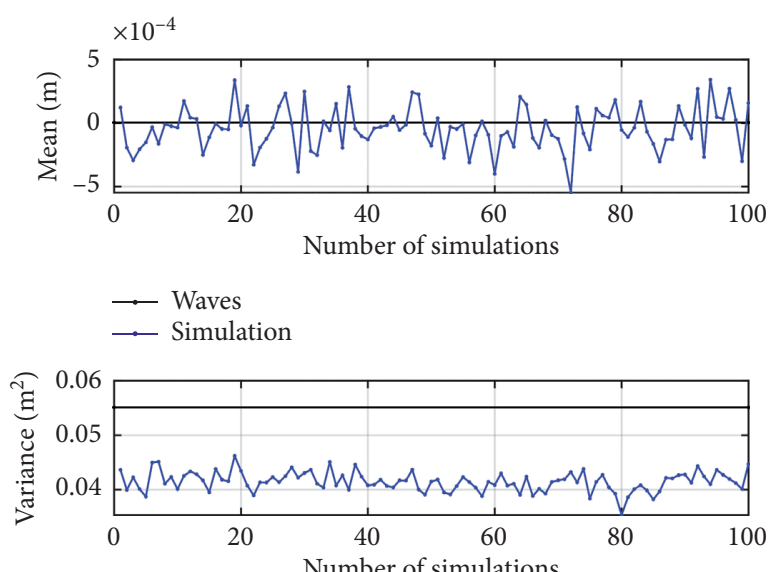

- - Waves

$\longrightarrow$ Simulation

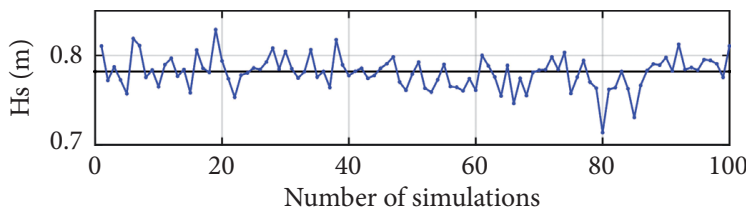

$\rightarrow$ Waves

$\longrightarrow$ Simulation

Figure 9: Comparison of statistical parameters.

the idea in Figure 4, and the wave information is extracted, as shown in Figure 12, according to equation (29).

As can be seen from the figure, the revised measurements are closer to the longitudinal trajectories of the waves without divergence, which is sufficient to estimate the sea state. Thus, this approach, which uses the virtual horizontal line to correct GNSS/SINS integrated data, performs well at extracting information in the Up direction.

4.3. Integrated Method When GNSS Breaks. The GNSS is an important means to correct the divergence of the SINS, and its combination with the SINS can figure wave height information well. However, when the severe sea conditions cause the GNSS to be intermittent for a long time, the results of the SINS will diverge because they cannot be corrected in
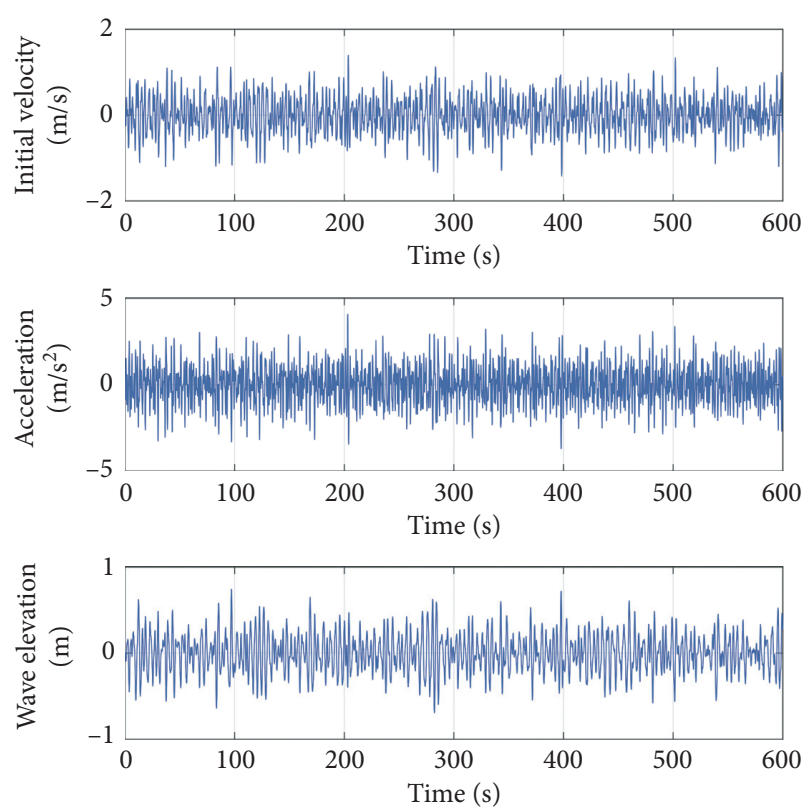

Figure 10: The simulated motion state.

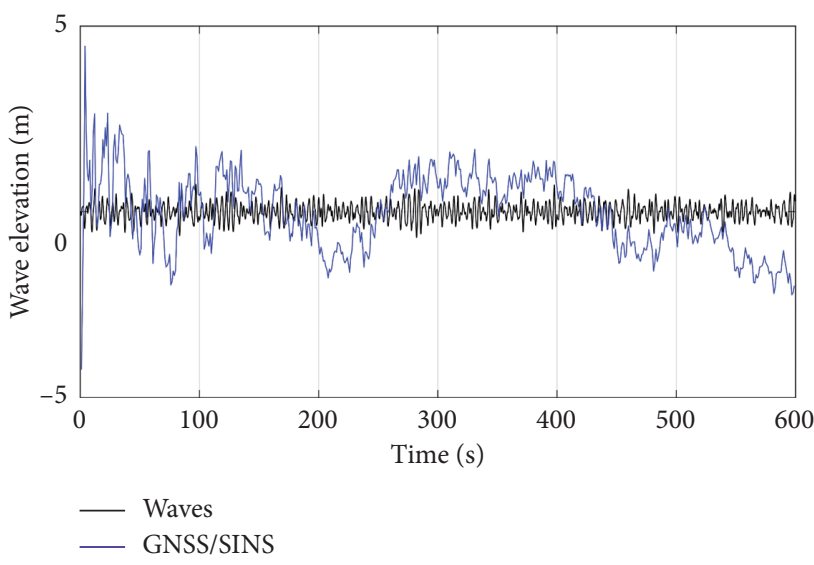

FigURE 11: GNSS/SINS positioning results in the up direction.

time. The wave height data simulated by wave spectrum can predict the waves in a short period statistically. Applying it to the measurement correction during GNSS interruption can 


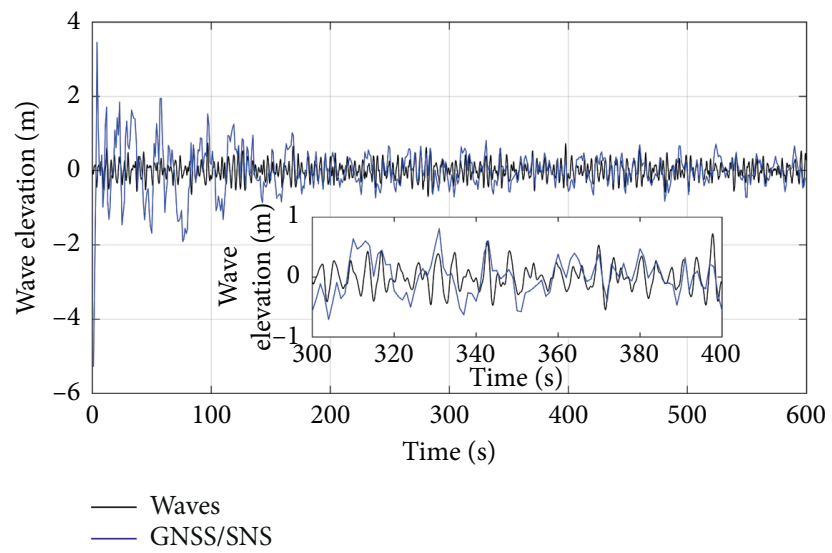

FIGURE 12: The corrected results from the GNSS/SINS integrated method.

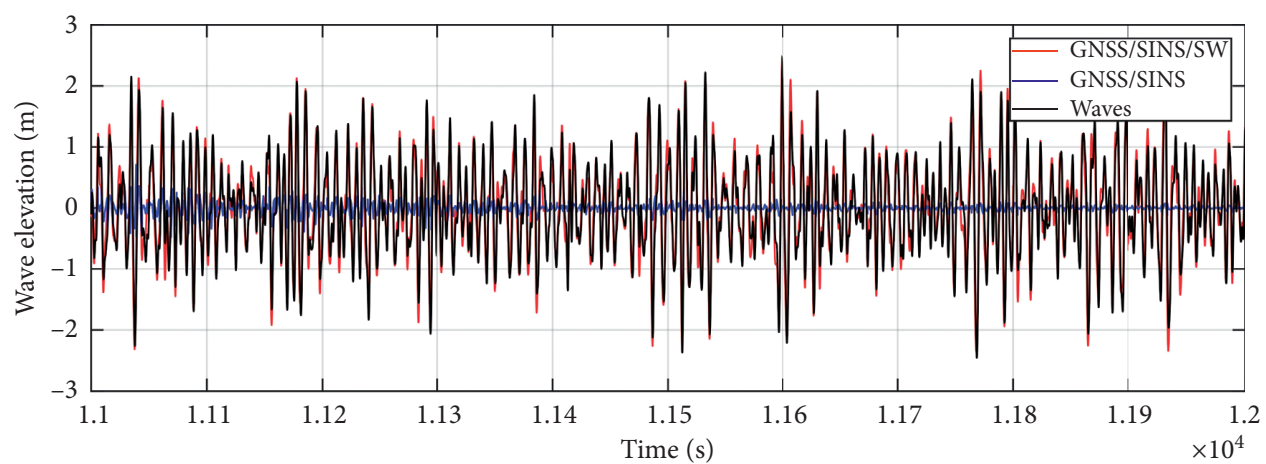

(a)

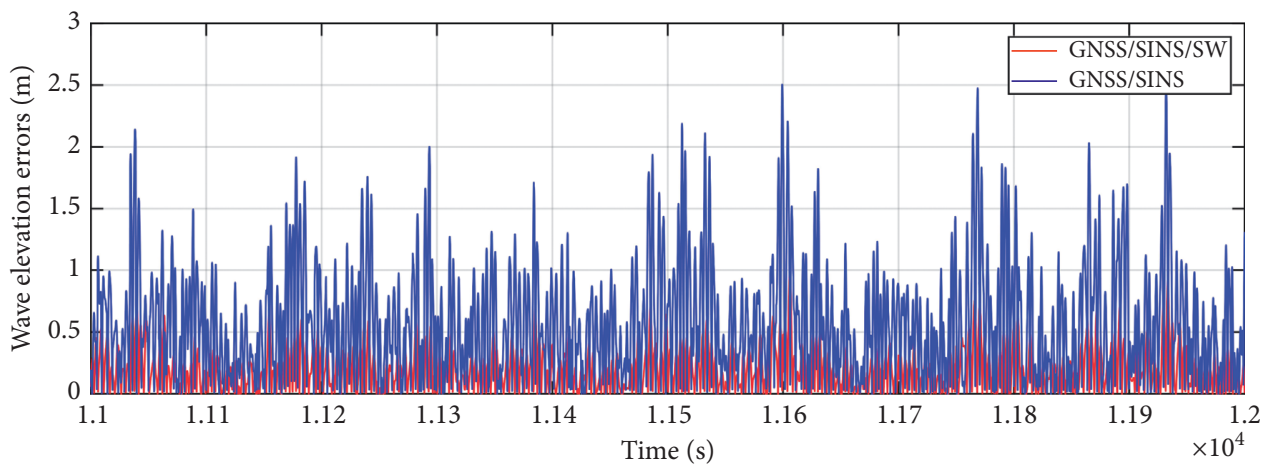

(b)

FIgURE 13: Advantages of SW applied in the integrated method during GNSS interruption.

provide a significant improvement effect, as shown in Figure 13.

As can be seen from Figure 13(a), compared with the reference value waves, the results of the GNSS/SINS integrated method did not perform well and could not obtain the wave height information. That is, because the interruption of the GNSS caused the SINS unable to be effectively corrected, the divergence of the SINS in the up direction gradually weakened the wave height information. The integrated method of GNSS/SINS/SW can fetch the wave height information very well, and the effect is better compared with the reference value. This is attributed to the good performance of SW in the Up direction during GNSS interruption, which made up for the deficiency of hardware caused by the GNSS failure with effective correction. The error comparison in Figure 13(b) further illustrates the effectiveness of the GNSS/SINS/SW method, whose error is far less than that of the GNSS/SINS method, which can be controlled at about 0.3 meters or less.

In order to show the excellent performance of the method in a more comprehensive way, the average wave heights $(\mathrm{AWH})$ and $\mathrm{SWH}$ are calculated every five minutes for ten hours, and the results are shown in Figure 14. 

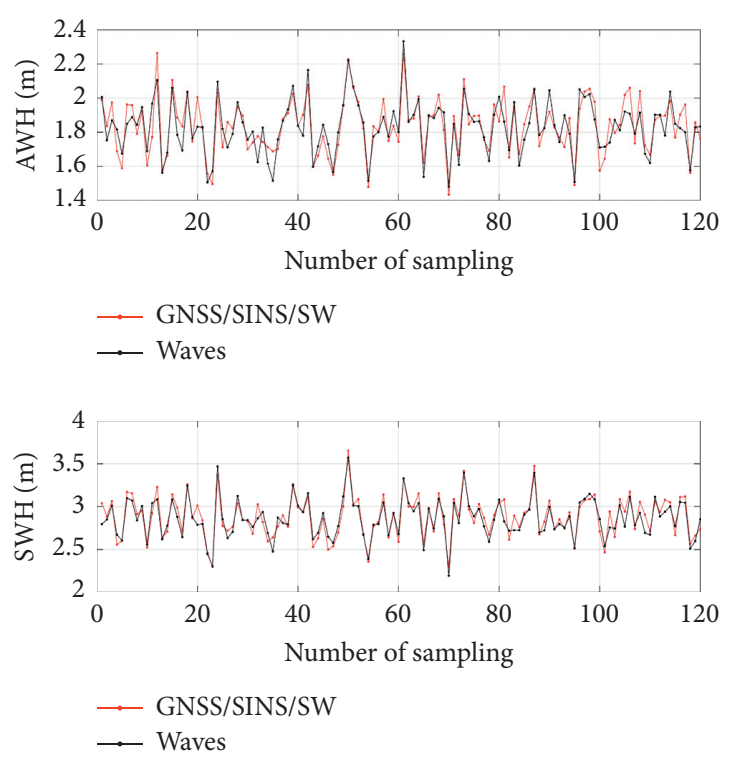

(a)
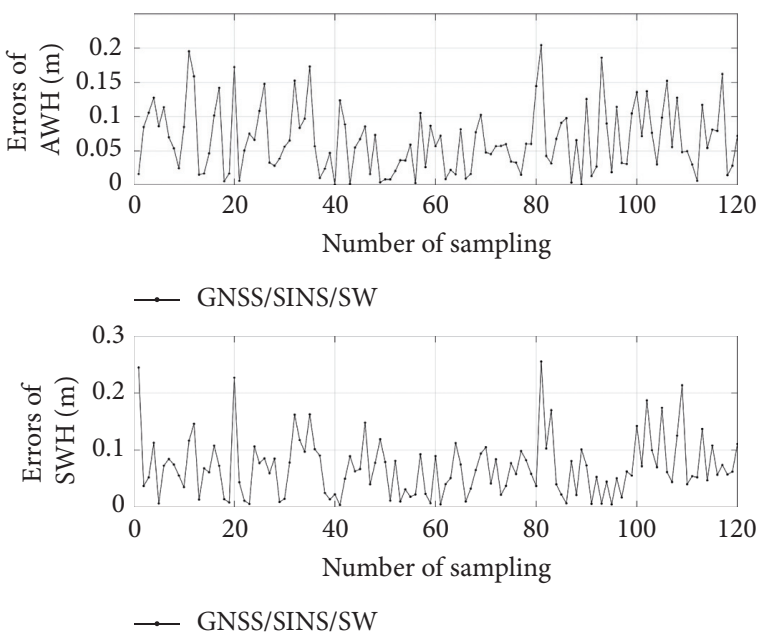

(b)

FIgURE 14: The performance of GNSS/SINS/SW in AWH and SWH.

AWH and SWH are two important parameters to estimate the sea state. Especially, in the application background of this paper, the staff can estimate the sea state based on this information and then make a judgment on whether it is suitable for the recycling work. According to the statistical results of 120 samples in Figure 14(a), AWH and SWH calculated by the GNSS/SINS/SW method has a certain difference between the calculated results and reference value at some sampling points, but the errors are still within the acceptable range. In general, $\mathrm{AWH}$ and SWH calculated by this method perform well overall, as can be seen intuitively in Figure 14(b). Their errors can be controlled at about 0.1 meters, and in some better performances, the errors are so small that can almost be ignored in the engineering application of this paper. The results can be used to estimate the sea state well and provide an effective reference for the recycling work.

\section{Conclusions}

Before recycling the detection equipment on the sea surface, the staff needs to know the sea state of its surrounding environment to determine whether it is suitable for operation. Without additional sensors, the sea state can be estimated intuitively by wave height measurement with the SINS and GNSS of the equipment such as AUVs and underwater gliders.

Under this application background, this paper proposes a wave correction method, which amends the results of the GNSS/SINS integrated method in the up direction by the virtual horizontal line. In addition, an integrated method of SINS and P-M spectrum is presented, which solves the problem of data divergence caused by GNSS interruption under bad sea conditions. The simulation results show that the problem is obviously improved, and the wave spectrum is of great significance in locating the sea-surface target.

\section{Data Availability}

The data required to reproduce these findings cannot be shared at this time as the data also form part of an ongoing study.

\section{Conflicts of Interest}

The authors declare that they have no conflicts of interest.

\section{Acknowledgments}

This work was supported by the National Natural Science Foundation of China (62073080 and 61921004).

\section{References}

[1] K. Komatsu and K. Tanaka, "Swell-dominant surface waves observed by a moored buoy with a GPS wave sensor in Otsuchi Bay, a ria in Sanriku, Japan," Journal of Oceanography, vol. 73, no. 1, pp. 87-101, 2017.

[2] H. Jiang, A. V. Babanin, and G. Chen, "Event-based validation of swell arrival time," Journal of Physical Oceanography, vol. 46, no. 12, 2016.

[3] S. C. Crosby, W. C. O’Reilly, and R. T. Guza, "Modeling long period swell in Southern California: practical boundary conditions from buoy observations and global wave model predictions," Journal of Atmospheric and Oceanic Technology, vol. 33, no. 8, 2016.

[4] H. Zhang, Q. Wu, and Ge Chen, "Validation of HY-2A remotely sensed wave heights against buoy data and jason-2 altimeter measurements," Journal of Atmospheric \& Oceanic Technology, vol. 32, no. 6, 2015.

[5] M. Jiang, $\mathrm{K} . \mathrm{Xu}$, and $\mathrm{Y}$. Liu, "Calibration and validation of reprocessed HY-2A altimeter wave height measurements using data from Buoys, Jason-2, Cryosat-2 and SARAL/ Altika," Journal of Atmospheric \& Oceanic Technology, vol. 35, no. 6, pp. 1331-1336, 2018. 
[6] Y. Tian, Z. Tian, J. Zhao, B. Wen, and W. Huang, "Wave height field extraction from first-order Doppler spectra of a dual-frequency wide-beam high-frequency surface wave radar," IEEE Transactions on Geoscience and Remote Sensing, vol. 58, no. 2, pp. 1017-1029, 2020.

[7] X. Liu, "Wave height estimation from ship-borne X-band nautical radar images," Journal of Sensors.vol. 2016, 2016.

[8] X. Liu, "Comparison of wave height measurement algorithms for ship-borne X-band nautical radar," Canadian Journal of Remote Sensors, vol. 42, no. 4, pp. 344-353, 2016.

[9] K. Yu, "Tsunami-wave parameter estimation using GNSSbased sea surface height measurement," IEEE Transactions on Geoscience and Remote Sensing, vol. 53, no. 5, pp. 2603-2611, 2015.

[10] Z. Tao, C. Liping, and L. Yao, "AUV underwater positioning algorithm based on interactive assistance of SINS and LBL," Sensors, vol. 16, no. 1, p. 42, 2015.

[11] D. Zhao, X. Liu, and H.J. Zhao, "Seamless integration of polarization compass and inertial navigation data with a selflearning multi-rate residual correction algorithm," Measurement, vol. 170, Article ID 108694, 2020.

[12] Q. Wang, C. S. Qian, and Z. J. Zhang, "Application of federated filter to AUV based on terrain-aided SINS," Applied Mechanics and Materials, vol. 711, pp. 338-341, 2015.

[13] T. Zhang and $\mathrm{X}$. Xu, "SINS/DVL/LBL interactive aiding positioning technology based on AUV," Conference RecordIEEE Instrumentation and Measurement Technology Conference, vol. 2015, pp. 745-750, 2015.

[14] G. Hu, S. Gao, and Y. Zhong, "A derivative UKF for tightly coupled INS/GPS integrated navigation," ISA Transactions, vol. 56, pp. 135-144, 2015.

[15] X. Zhao, J. Li, and X. Yan, "Robust adaptive cubature kalman filter and its application to ultra-tightly coupled SINS/GPS navigation system," Sensors, vol. 18, no. 7, 2018.

[16] X. Xu, D. Xu, and T. Zhang, "In-motion coarse alignment method for SINS/GPS using position loci," IEEE Sensors Journal, vol. 10, p. 1, 2019.

[17] C. Kai-Wei, L. Cheng-An, and K. Chung-Yen, "A feasibility analysis of land-based sins/gnss gravimetry for groundwater resource detection in Taiwan," Sensors, vol. 15, no. 10, pp. 25039-25054, 2015.

[18] G. Hu, B. Gao, Y. Zhong, and C. Gu, "Unscented kalman filter with process noise covariance estimation for vehicular ins/gps integration system," Information Fusion, vol. 64, pp. 194-204, 2020.

[19] C. Shen, Y. Zhang, and J. Tang, "Dual-optimization for a MEMS-INS/GPS system during GPS outages based on the cubature Kalman filter and neural networks," Mechanical Systems and Signal Processing, vol. 133, Article ID 106222, 2019.

[20] D. Wang, X. S. Xu, and Y. Q. Yao, "A novel SINS/DVL tightly integrated navigation method for complex environment," IEEE Transactions on Instrumentation and Measurement, vol. 69, no. 7, pp. 5183-5196, 2019.

[21] G. Hu, W. Wang, Y. Zhong, B. Gao, and C. Gu, "A new direct filtering approach to INS/GNSS integration," Aerospace Science and Technology, vol. 77, pp. 755-764, 2018.

[22] S. Q. Wang and B. C. Liang, Wave Mechanics for Ocean Engineering, Qingdao: China Ocean University Press, Qingdao, China, 2013.

[23] L. Shouhua, J. Ning, and G. Changlong, "A proposed frequency spectrum for fully developed wind waves by revisiting P-M spectrum," Journal of Ocean University of China, vol. 10, no. 4, pp. 331-335, 2011. 